\title{
Online Laboratory Experiment Learning Module for Biomedical Engineering Physiological Laboratory Courses
}

\author{
Christine E. King @i, Casey Trevino, and Piyashi Biswas \\ Department of Biomedical Engineering, University of California Irvine, Irvine, CA, USA
}

(Received 20 June 2020; accepted 30 September 2020; published online 16 October 2020)

\begin{abstract}
By early spring 2020, the COVID-19 pandemic caused mandatory campus closures of academic institutions nationwide, prompting the rapid transition to online instruction. While lectures and exams were more straightforwardly administered online using video-chatting software, many hands-on laboratory-based courses were forced to develop creative solutions. In response to online instructional requirements, instructors at the University of California Irvine developed an online electroencephalography (EEG) laboratory to simulate the laboratory experiment for students unable to perform the experiment on campus. The laboratory experiment was performed and video recorded by the instructional team under three different scenarios to provide students with multiple data sets acquired under various experimental conditions often enacted by students. Students were required to complete a pre-lab quiz, analyze the acquired EEG data offline, complete a post-lab quiz, and submit their laboratory report to communicate their findings prior to final exams. Student performances compared to prior student performances, and qualitative survey responses, were examined to assess the effectiveness of and response to the online laboratory format. Based on student feedback and lab report grades, the majority of students responded positively and demonstrated an understanding of the EEG experiment's learning outcomes. In summary, the online EEG laboratory enabled students to achieve the main learning objectives and become familiar with the laboratory experiment, indicating its success as an alternative laboratory experiment.
\end{abstract}

Keywords-Online laboratory courses, Online laboratories, Electroencephalogram (EEG), Undergraduate education, COVID-19 education.

Address correspondence to Christine E. King, Department of Biomedical Engineering, University of California Irvine, Irvine, CA, USA. Electronic mail: kingce@uci.edu

\section{CHALLENGE STATEMENT}

In the era of the COVID-19 pandemic, access for students to be able to perform in-person laboratory experiments has become increasingly difficult. ${ }^{1} \mathrm{Com}-$ bined with rapidly increasing biomedical engineering class sizes across the country, opportunities for undergraduate students to experience in person laboratory experiments will become scarce if not impossible.

In order to allow all students the opportunity to learn how to perform physiological experiments for biomedical engineering applications remotely, online courses must be provided to students to be able to perform experimentation while practicing social distancing and to meet necessary learning outcomes for biomedical engineers. ${ }^{6}$ As a result of the challenges introduced due to the COVID-19 pandemic, a number of institutions within the Biomedical Engineering (BME) community compiled an online repository to share knowledge and guidance to implement online learning curricula. ${ }^{1}$ Furthermore, a number of investigators have analyzed the diverse responses to the complex challenges to support knowledge-sharing activity across universities. ${ }^{4}$ While institutions rapidly transitioned various in-person laboratory courses to online solutions, a multitude of online laboratories have already been established to supplement or substitute student instruction, providing easy access to experiments for all students, and reducing cost efficiency without reducing the quality of learning (Online Appendix A). ${ }^{15}$ Online learning has been shown to be as effective as in person learning with added advantages, supporting its use as an alternative teaching 
method during the period of the COVID-19 pandemic. $^{11}$

Although online learning environments facilitate easy access to students unable to attend in-person laboratories, remote learning introduces a new set of particular challenges to consider when developing home-based learning programs. ${ }^{12}$ In short, the best practices recommend emphasizing equity for students with special learning circumstances, with limited access to devices for online learning, and who live in noisy environments or overseas in different timezones not conducive to synchronous learning. ${ }^{9,12}$ Compounding these obstacles, effective online instruction is often inhibited by low concentration from students, decreased faculty and student interaction, and decreased student participation. ${ }^{2}$

One of the undergraduate BME design courses at the University of California Irvine, which required conversion to online instruction is the Biomedical Engineering Laboratory. The curriculum involves an electroencephalography (EEG) lab to introduce students to measurement and analysis of biological systems using engineering tools and techniques. Prerequisites of this course included courses such as Sensory Motor Systems, Quantitative Physiology: Organ Transport Systems, Biomedical Signals and Systems, and Design of Biomedical Electronics, as this laboratory is a required upper division course for all BME undergraduate students. Because of social distancing requirements and the resulting campus closure, ${ }^{16}$ the EEG lab was converted to an online laboratory to meet the same learning objectives that would have been provided in person without reducing educational quality, student engagement, and performance. Here, we share and reflect upon the approach used to develop a online EEG laboratory, given the time constraints from the campus closure, and an assessment of the effectiveness of this effort based on student feedback and performance.

\section{NOVEL INITIATIVE}

Just prior to the mandatory shutdown of all teaching facilities, the instructional team performed the laboratory experiment under three different scenarios that have been frequently observed by the professor in prior years. These were conducted given the course learning objectives met by this laboratory section, which include ${ }^{6}$ :

- An ability to communicate effectively with a wide range of audiences (SO 3)

- An ability to develop and conduct appropriate experimentation, analyze and interpret data, and use engineering judgement to draw conclusions ( $\mathrm{SO}$ 6)

Although the novel online video structure of the course was able to accomplish SO 3 and partially SO 6 , the ability to conduct appropriate experimentation that students would have been able perform in person had to be given via video scenarios that attempted to highlight the importance of appropriate experimentation for physiological laboratories, such as the data acquisition settings or reduction of noise prior to data collection. Instances such as these have been frequently observed by prior teaching assistants and instructors of the course, which were the focus of the video scenarios so that students could experience similar settings as inperson, and interpret the data using engineering and statistical approaches to draw upon conclusions.

The three scenarios presented to the students included a thorough experiment where all protocol procedures were followed correctly, one where the experimenter "forgets" key techniques and procedures, and one where there was no regard to the procedures and hardware settings. These video experiments corresponded to EEG data of high, mediocre, and poor quality, which allowed students to learn the key components associated with the laboratory as if they were to perform the experiment in person.

\section{EEG Laboratory Experiment}

The EEG laboratory experiment focused on measuring the alpha rhythm. ${ }^{7}$ Originally observed by Dr. Hans Berger, ${ }^{8}$ the alpha rhythm $(8-12 \mathrm{~Hz})$ is an increase in EEG power predominantly over the occipital lobe when a person is relaxed and has their eyes closed. When the person is engaged in a mental task or opens their eyes, the alpha rhythm becomes desynchronized and the power in this band decreases. Students were tasked to measure these signals and verify this phenomenon through signal analyses of EEG data acquired by the instructional team under each scenario (see Online Appendix A for full details of the protocol and Online Appendix B for the corresponding lecture material). The protocol and analysis utilized MATLAB (MathWorks, Natick, MA), and data were acquired using a BIOPAC data acquisition system and AcqKnowledge data collection software (Biopac Systems Inc., Goleta, CA). In addition, as seen in Fig. 1 below, the EEG was collected from four channels of a 19-channel Neuroscan Quik-Cap (Compumedics Ltd., Victoria, Australia) for each scenario.

During the in-person laboratory experiment, the students would collect approximately $10 \mathrm{~min}$ of EEG data, alternating every $10-30 \mathrm{~s}$ between opening and closing their eyes during relaxation. In lieu of provid- 
ing this choice online, the students were given the below trials and duration of time per trial (Table 1), after being encouraged during the lecture to consider sample sizes as well as physiological limitations of the subject (e.g., paying attention, drowsiness).

Students were provided three datasets corresponding to each scenario; however, they were not informed of the varying quality of each separate data collection. Students were instructed to analyze all three scenarios and write a complete laboratory report on their findings and observations, describing why they obtained different results from each scenario in the discussion and results section based on how data were collected. In particular, Online Appendix $\mathrm{C}$ describes the grading guidelines in detail, where students were asked to meet the following course learning outcomes:

- The ability to effectively communicate professionally to a wide range of audiences

- The ability to understand and describe the concepts regarding the scientific and physiological principles of the laboratory experiment

- Ability to describe important software, hardware, statistical methods, experimental protocols and procedures in

- Present data appropriately to a technical audience with no prior knowledge of EEG

- Summarize the main results found, and draw conclusions given the findings.
Visual Processing of the Experiments

The scenarios were filmed by the instructional team just prior to campus shutdown. The filming was conducted quickly with limited resources, as the team only had access to a Nikon CoolPix P900 camera (Nikon, Minato City, Tokyo, Japan). To accommodate for the poor filming conditions that were presented, the professor used Camtasia (TechSmith Corporation, Okemos, Michigan, USA) to significantly edit the scenario videos. As seen in Table 2 below, the videos, corresponding data, and analyses code are provided as hyperlinks for other instructors and institutions to easily adopt.

The videos were edited to include improvements to the audio, sped up during less important portions of the experimental procedure, and annotations were added to highlight important information and procedures during the experiments. As seen in Fig. 2 below, annotations were used to describe what the students were observing in terms of hardware and experimental setup, signal quality observed during the experiment, and appropriate procedures such as those performed after the experiment is completed.

\section{Online Laboratory Structure of the Experiments}

Typically, the Biomedical Engineering Laboratory is a 10 week course in which students attend lectures, perform the EEG laboratory data collection, analyses,

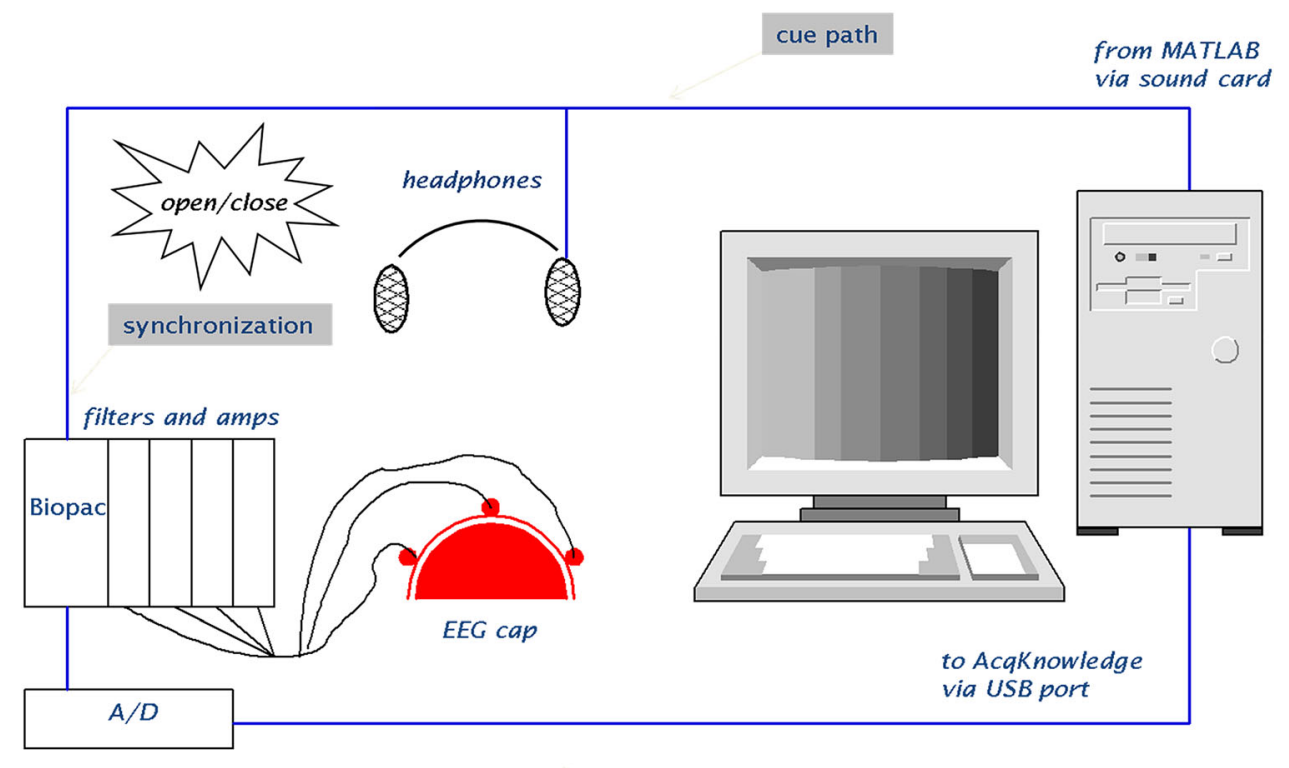

data acquisition path

FIGURE 1. Overall experimental hardware setup of the EEG laboratory. 
TABLE 1. Number of trials and duration of each trial for each scenario presented during the online EEG laboratory experiment.

\begin{tabular}{lcc}
\hline Scenario & Number of Trials & Duration of trial per sample (s) \\
\hline Scenario 1-protocols performed appropriately & 10 & 20 \\
Scenario 2-few protocols missing, mediocre conditions & 10 & 20 \\
Scenario 3-most protocols ignored, poor conditions & 5 & 10 \\
\hline
\end{tabular}

TABLE 2. Hyperlinks of the videos, corresponding data, and analyses code for the EEG laboratory portion of the course.

\begin{tabular}{|c|c|}
\hline Scenario & Hyperlink \\
\hline Master directory folder & $\begin{array}{l}\text { Master directory link } \\
\text { https://drive.google.com/drive/folders/1qTQYu-V-IKnpwtmhMSeky8VheAkLOIq-?usp= } \\
\text { sharing }\end{array}$ \\
\hline Introduction & $\begin{array}{l}\text { Video link (length-0:09) } \\
\text { https://drive.google.com/file/d/1EgAH4cxi-4Yf2Gues2RxMT_uqyB16LBE/view?usp=s } \\
\text { haring }\end{array}$ \\
\hline Scenario 1: protocols performed appropriately & $\begin{array}{l}\text { Scenario } 1 \text { Video (length-29:15) } \\
\text { https://drive.google.com/file/d/1nybT6AHQvPQ3DQIwh9B6izS-Txb9EWsS/view?usp= } \\
\text { sharing } \\
\text { Scenario } 1 \text { Data } \\
\text { https://drive.google.com/file/d/1Zr3SKQWy-rSi1xt-uTuGw04mdVHVJdYx/view?usp=s } \\
\text { haring }\end{array}$ \\
\hline $\begin{array}{l}\text { Scenario 2: few protocols missing, mediocre con- } \\
\text { ditions }\end{array}$ & $\begin{array}{l}\text { Scenario } 2 \text { Video (length-8:33) } \\
\text { https://drive.google.com/file/d/13C9lqJdp3FyA4qzxuVnglht7V_ZBDVyh/view?usp=sh } \\
\text { aring } \\
\text { Scenario } 2 \text { Data } \\
\text { https://drive.google.com/file/d/16L_YBfQUCbZ2qq-DMtBj8btoyPCQ8HXy/view?usp=s } \\
\text { haring }\end{array}$ \\
\hline Scenario 3: most protocols ignored, poor conditions & $\begin{array}{l}\text { Scenario } 3 \text { Video (length-4:28) } \\
\text { https://drive.google.com/file/d/1-uMJoNFQxLcVjOhQ_w8Mxh7wnlsk-MQW/view?usp= } \\
\text { sharing } \\
\text { Scenario } 3 \text { Data } \\
\text { https://drive.google.com/file/d/10YE6qdpct2qXOJEIY6h4kmRI20u9GX5n/view?usp=s } \\
\text { haring }\end{array}$ \\
\hline Data analyses code & $\begin{array}{l}\text { Code } \\
\text { https://drive.google.com/file/d/1pdtQfoOPZ62rBIS_4ONJXn3f4z7iJDp8/view?usp=sha } \\
\text { ring }\end{array}$ \\
\hline
\end{tabular}

and reflection through a scientific written report over the course of 5 weeks; then, students perform a biomolecular testing laboratory over the course of the last 5 weeks in a similar format. When these labs were conducted in person, student groups rotated through the laboratories due to the limited laboratory stations and large class size. Due to the transition to an online environment during the COVID-19 pandemic, the video experiment format allowed the instructors to improve their courses by adding additional topics and assessments (Table 3). For instance, for the EEG laboratory portion of the course, pre- and post-lab quizzes (Online Appendix D) were added to guide students on the major procedures and protocols required for experimentation, and why they are performed. Addi- tionally, required reference and texts and documents were included in the EEG laboratory, which allowed students to further understand the laboratory practices, science, and BME applications of EEG, such as brain-computer interfaces.

\section{Assessment of the Online Laboratory}

To assess the feasibility and acceptability of the online EEG laboratory using the scenario videos, a short survey was sent to the students. All students that participated in the survey were provided with informed consent (University of California Irvine IRB Approval Number: 2018-4211). To this end, the following survey questions were posed:

\section{BMES



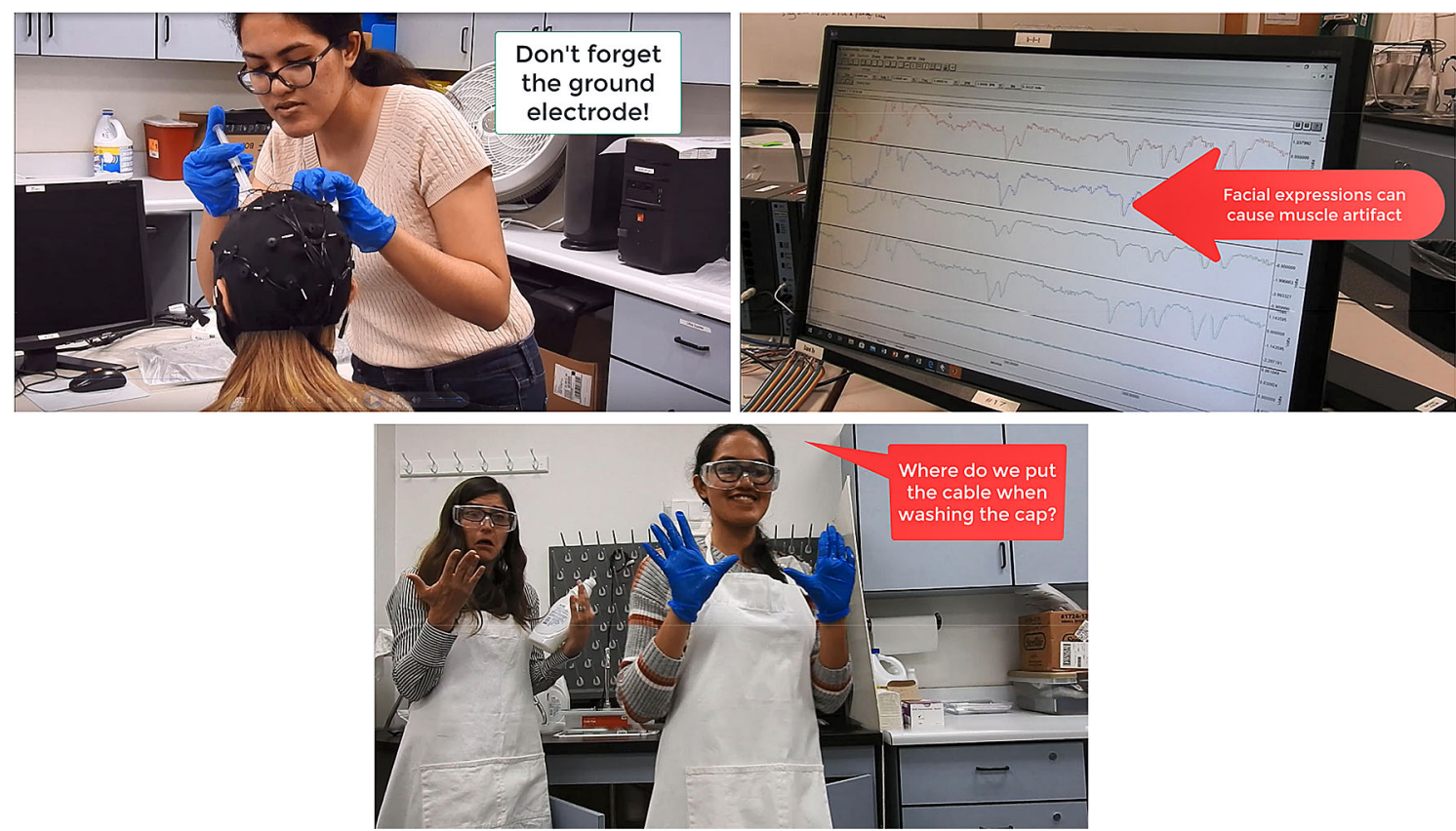

FIGURE 2. Screenshots of different portions of the laboratory experiment scenarios showing annotations during hardware procedures (top left), software procedures (top right), and the post-experiment cleaning procedure (bottom).

TABLE 3. Overall structure of the EEG laboratory portion of the Biomedical Engineering Laboratory course.

Week of the EEG Laboratory Course

Week 1: Introduction to EEG Lab and Pre Lab Training

Week 2: Introduction to Molecular Diagnostics Lab and Finish Pre Lab Training

Week 3: EEG Lab Instructions and Pre-Lab Quiz

Week 4: EEG Lab Video

Week 5: EEG Post-Lab Quiz and Lab Report

Week 6: EEG Conclusion and Reference Text Required Reading

Week 6-10: Biomolecular Online Laboratory Module

Week 10: EEG Laboratory Review Lecture

Finals Week: Final Exam

- What worked well during the videos?

- What were your reactions to the lab run-through videos?

- What did you like?

- What would you have done differently?

\section{REFLECTION}

The main goal of this research was to provide an alternative to hands-on lab research during the COVID-19 crisis using online lab settings. The objectives outlined in this course were to learn concepts in Electroencephalogram measurement, become familiar with the EEG laboratory setup and experimental procedure, collect and analyze data using statistical tests, and reflect on the impact of following correct protocol on observed results. The course incorporated the strategies of the "Five E Cycle" as suggested by Bybee et al. ${ }^{3}$ The "Engagement" involved an introductory interactive remote lecture and pre-lab training. For "Exploration", the students thoroughly read the lab instruction manual and attempted a pre-lab quiz to establish the concepts (Table 3). The detailed demonstration videos of three scenarios provided them with "Explanation" of the methods and possible mistakes, ending with the post-lab quiz. These concepts were "Elaborated" by analyzing the three sets of data with varying qualities based on the staged scenarios and a written lab report. Finally, this report was "Evaluated" in a set rubric along with the quizzes and a multiple-choice final examination component.

The students performed significantly better in the evaluative components as compared to the previous 
year (Fig. 3). The final exam evaluates the students' conceptual understanding of EEG measurement, experimental procedure, and statistical analysis while the lab report evaluates their individual statistical analysis of the collected EEG data and reflection of the reasons for the observed results. The primary objective we were unable to evaluate was the quality of the data collection, typically demonstrated through individual lab reports. A secondary objective to incorporate teamwork could also not be achieved through this version of the course. Based on the performance, we conclude that despite the lack of hands-on experience, students were able to achieve four out of five objectives outlined in the course. The inherent lack of hands-on experience and data collection was tackled by including the staged-scenario based approach. This novel approach helped students learn about the possible

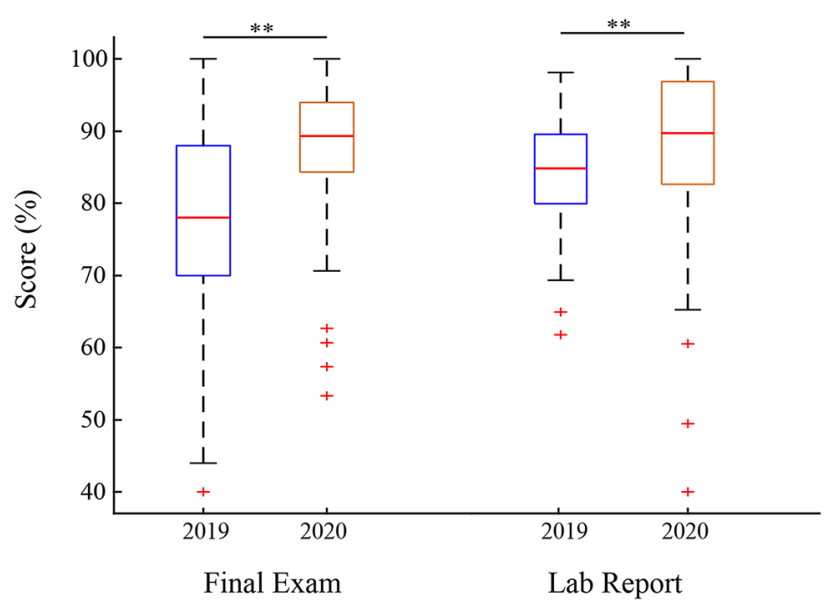

FIGURE 3. Student performance compared to the previous year based on final exam and lab report scores. Note that the same exam questions and lab report rubrics were used across both years. mistakes and analyze their impact on the recorded data. This provided a unique opportunity to understand the reasoning behind the protocol and highlighted the importance of good practices. Such scenario-based learning has been implemented previously in online laboratories (Online Appendix A).

To assess the quality of implementation and achievement of desired outcomes, students were surveyed and teaching assistants (TAs) were interviewed at the end of the course, as described in the Assessment of the Online Laboratory section above. Thirty-five students responded to the voluntary post-lab survey; the qualitative responses are summarized in Table 4. The responses provided constructive feedback for the development of this course. The all-round access to videos allowed students to work at their own pace and watch the experiment being conducted multiple times. This helped them take more detailed notes as compared to a live experiment. The important aspects of experimentation and practical details were highlighted in videos which helped students understand concepts and learn about common mistakes to avoid. This also gave students a chance to learn correct techniques which would be difficult in 3-h lab sessions with multiple groups performing the experiment simultaneously. The lab report performances revealed that only 2-3 students of 100 had trouble understanding the experiments. The TAs and the professor were available for contact via email which let the students reach out personally with questions and hence received individual attention for their doubts.

Based on the survey responses, some shortcomings have been identified. The main criticism was about the video quality (lack of a cameraperson, unclear audio and unavailable screen recording). Some students mentioned difficulty in engaging with longer videos like Scenario 1 (Table 2). These issues will be addressed in

TABLE 4. Qualitative assessment of student survey responses

\begin{tabular}{lcll}
\hline Category & Positive responses (\%) & Negative responses (\%) & No response (\%) \\
\hline Video annotations (Q\&A, commentary) & 77.1 & 0 & 22.9 \\
Video quality (audio, visual, direction) & 11.4 & 62.9 & 25.7 \\
Informative & 37.2 & 0 & 62.8 \\
Efficiency and entertaining & 34.3 & 0 & 65.7 \\
Narration of procedures & 34.2 & 0 & 65.8 \\
Three scenarios & 25.7 & 5.7 & 68.6 \\
Step-by-step procedure & 17.2 & 0 & 82.8 \\
Online laboratory replacement & 0 & 11.4 & 88.6 \\
\hline
\end{tabular}

Categories (left) summarize the general classification of the survey responses based on the free-response format. The percentage of positive (middle) and negative (right) responses are computed based on the number of positive or negative reactions to the corresponding category divided by the total number of participants $(n=35)$. Of the 8 categories, 6 had mostly positive reactions while two were mostly negative. Categories (left) summarize the general classification of the survey responses based on the free-response format. The percentage of positive (middle) and negative (right) responses are computed based on the number of positive or negative reactions to the corresponding category divided by the total number of participants $(n=35)$. Of the 8 categories, 6 had mostly positive reactions while two were mostly negative. 
future implementations of the course by addition of transcripts and captions, professional video recording, and guidebooks to use the online material more efficiently. Further, the demo videos will be shortened to snippets of less than $10 \mathrm{~min}$, and Camtasia will be used to add small quizzes throughout the videos to increase engagement. Students will also be required to submit the code used for the data analysis to ascertain originality of work. The inclusivity of the course can be improved by ADA compliant technology for people with audio and visual impairments. These improvements will be provided to other instructors in the future and the files can be accessed using the same master directory links provided in Table 2 above. Interested instructors can sign up for updates using this hyperlink: https://forms.gle/A14WyShpDCGHqr6S8. To elevate the impact of online laboratory settings to be used as a permanent replacement, 3D virtual reality environments can be used to create a space for students to conduct EEG experiments. An ongoing project being conducted at the instructor's laboratory involves using two $180^{\circ}$ cameras to film the laboratory to simulate students entering the lab remotely to conduct experiments. Interactive game-like scenarios could be included to remind them of proper protocol. Teamwork can be fostered through lab sessions with multiple participants. This method would incorporate the advantages of both online and hands-on laboratories.

The success of this online laboratory opens more opportunities for labs to adapt to situations that require distance learning courses. It has been shown by multiple previous studies that online laboratories can improve student learning from in-person labs serving as a supplementary component..$^{5,10,13,14}$ The online EEG laboratory provided students with an immediate alternative to the traditional laboratory course to adapt to the COVID-19 crisis. While students did not have the opportunity to experience hands-on lab experiments, they were able to successfully achieve the main learning objectives of the course. We are confident that the inclusion of advanced technology like virtual reality in classrooms can lead to more efficient student learning and be present as a ready alternative for any future crisis that would prevent in-person education.

\section{ELECTRONIC SUPPLEMENTARY MATERIAL}

The online version of this article (https://doi.org/10. 1007/s43683-020-00034-9) contains supplementary material, which is available to authorized users.

\section{ACKNOWLEDGMENTS}

We would like to acknowledge James Shyu of the Department of Biomedical Engineering at the University of California Irvine for his assistance in preparing the laboratory experiment videos.

\section{DATA AVAILABILITY}

All de-identified survey data will be made available by contacting the corresponding author, Dr. Christine King, kingce@uci.edu. All data associated with the laboratory module is available in the main body and appendices of the manuscript.

\section{CODE AVAILABILITY}

All software and code is made available in the main body of the manuscript.

\section{CONSENT TO PARTICIPATE}

All participants in this study were provided with informed consent (University of California Irvine IRB Approval Number: 2018-4211). The authors transfer to BMES the non-exclusive publication rights and warrant that the authors' contribution is original and that the authors have full power to make this grant. The authors accept responsibility for releasing this material. This transfer of publication rights covers the non-exclusive right to reproduce and distribute the article, including reprints, translations, photographic reproductions, microform, electronic form (offline, online) or any other reproductions of similar nature.

\section{CONSENT FOR PUBLICATION}

The authors transfer to BMES the non-exclusive publication rights and warrant that the authors' contribution is original and that the authors have full power to make this grant. The authors accept responsibility for releasing this material. This transfer of publication rights covers the non-exclusive right to reproduce and distribute the article, including reprints, translations, photographic reproductions, microform, electronic form (offline, online) or any other reproductions of similar nature.

\section{CONFLICT OF INTEREST}

The authors declare that they have no competing interests. 


\section{ETHICAL APPROVAL}

This study was approved by the University of California Irvine Institutional Review Board, Approval Number: 2018-4211.

\section{REFERENCES}

${ }^{1}$ Amos JR, Howard C. How social distancing brought us closer as a BME community. Ann Biomed Eng. 2020. h ttps://doi.org/10.1007/s10439-020-02501-4.

${ }^{2}$ Bao W. COVID-19 and online teaching in higher education: a case study of Peking University. Hum Behav Emerg Technol. 2020. https://doi.org/10.1002/hbe2.191.

${ }^{3}$ Bybee RW. The BSCS 5E instructional model and 21st century skills. Colorado Springs: BSCS; 2009.

${ }^{4}$ Crawford J, Butler-Henderson K, Rudolph J, Glowatz M. COVID-19: 20 countries' higher education intra-period digital pedagogy responses. J Appl Teach Learn. 2020. h ttps://doi.org/10.37074/jalt.2020.3.1.7.

${ }^{5}$ Darrah M, Humbert R, Finstein J, Simon M, Hopkins J. Are virtual labs as effective as hands-on labs for undergraduate physics? A comparative study at two major universities. J Sci Educ Technol. 2014. https://doi.org/10.1007/ s10956-014-9513-9.

${ }^{6}$ Engineering Accreditation Commission. Criteria for accrediting engineering programs 2018-2019. Baltimore: Accreditation Board for Engineering and Technology (ABET) Inc; 2018.

${ }^{7}$ Fisch BJ, Spehlmann R. Fisch and Spehlmann's EEG primer: basic principles of digital and analog EEG. Amsterdam: Elsevier Health Sciences; 1991.

${ }^{8}$ Gloor P. Hans Berger on electroencephalography. Am J EEG Technol. 1969. https://doi.org/10.1080/00029238.196 9.11080728 .
${ }^{9}$ Moore R, Vitale D, Stawinoga N. The digital divide and educational equity: a look at students with very limited access to electronic devices at home. Insights in Education and Work. ACT, Inc.; 2018.

${ }^{10}$ Morozov M, Tanakov A, Gerasimov A, Bystrov D, Cvirco E. Virtual chemistry laboratory for school education. In: IEEE International Conference on Advanced Learning Technologies, 2004. Proceedings. https://doi.org/10.1109/I CALT.2004.1357486.

${ }^{11}$ Pei L, Wu H. Does online learning work better than offline learning in undergraduate medical education? A systematic review and meta-analysis. Med Educ Online. 2019;24(1):1666538. https://doi.org/10.1080/10872981.2019 .1666538 .

${ }^{12}$ Reich J, Buttimer CJ, Fang A, Hillaire G, Hirsch K, Larke LR, Littenberg-Tobias J, Moussapour RM, Napier A, Thompson M, Slama R. Remote learning guidance from state education agencies during the covid-19 pandemic: a first look. https://doi.org/10.35542/osf.io/437e2.

${ }^{13}$ Rowe RJ, Koban L, Davidoff AJ, Thompson KH. Efficacy of online laboratory science courses. J Form Des Learn. 2018. https://doi.org/10.1007/s41686-017-0014-0.

${ }^{14}$ Scheckler RK. Virtual labs: a substitute for traditional labs? Int J Dev Biol. 2003;47(2-3):231-6.

${ }^{15}$ Verawardina U, Asnur L, Lubis AL, Hendriyani Y, Ramadhani D, Dewi IP, Darni R, Betri TJ, Susanti W, Sriwahyuni T. Reviewing online learning facing the covid19 outbreak. J Talent Dev Excell. 2020;12(3s):385-92.

${ }^{16}$ Viner RM, Russell SJ, Croker H, Packer J, Ward J, Stansfield C, Mytton O, Bonell C, Booy R. School closure and management practices during coronavirus outbreaks including COVID-19: a rapid systematic review. Lancet Child Adolesc Health. 2020. https://doi.org/10.1016/s23524642(20)30095-x.

Publisher's Note Springer Nature remains neutral with regard to jurisdictional claims in published maps and institutional affiliations. 